\title{
Some disguises of attention deficit hyperactivity disorder
}

\section{H Perera $^{1}$ and MDI Amerasinghe Waas ${ }^{2}$}

(Index words: Compulsive water drinking and salt eating, foreign body insertion in vagina, self-harm)

\section{Introduction}

The essential features of attention deficit hyperactivity disorder (ADHD) are persistent hyperactivity, attention deficit and impulsiveness recognisable from early childhood. These symptoms are pervasive to many settings such as the classroom, home and elsewhere [1].
Inattention and hyperactivity cause impairment of social behaviour and learning. Parents and teachers often seek help for children with ADHD for underachievement in school work and disruption of classroom activities. Impulsiveness generally manifests as impatience, aggression, lack of understanding about safety and

${ }^{1}$ Department of Psychological Medicine, University of Colombo and ${ }^{2}$ Lady Ridgeway Hospital for Children, Colombo, Sri Lanka.

Correspondence: HP; e-mail: <hemamalip@yahoo.com> (Competing interests: none declared). Received 17 June 2005 and accepted 21 June 2005. 
proneness to accidents. Hence children with ADHD are seen in accident and emergency and surgical settings for head trauma, fractures, burns and other non-intentional injuries [2-4]. Then the primary problem of ADHD could go unrecognised as the treatment of injuries takes priority, and parents may fail to report the pre-existing behaviour problems.

We report four children with ADHD whose primary diagnosis went unrecognised because of their unusual clinical presentations. The objective of reporting these cases is to discuss the possible mechanism for the presence of such symptoms in a child with ADHD. The diagnosis of ADHD was made in all the cases using DSM IV criteria [1], further validated by observation of behaviour during an inpatient period and from scores obtained in a standardised diagnostic tool. In addition, other psychometric tests were used to assess the level of attention deficit and learning difficulties.

\section{Case1 : The compulsive water drinker}

This 10-year old boy was initially admitted for investigation of excess water drinking and frequent voiding of urine of 2 years' duration. His mother believed that the frequent voiding was causing poor weight gain. In hospital, the investigations were mainly directed at possible diagnoses of diabetes mellitus, diabetes insipidus or a cerebral tumour, but none were found. He was then referred for psychological management. He constantly sipped water from a bottle when at home and in the classroom, and interrupted lessons several times to go to the toilet. There were complaints from the class-teacher about restlessness, difficulty in keeping him seated and poor school performance, which antedated the current symptoms. He was also taking sodium valproate for epilepsy. Treatment with methylphenidate produced dramatic results in reducing the excess water intake and polyuria. On follow up, he had two recurrences of the behaviour when methylphenidate was stopped and remissions on reintroduction of the drug.

\section{Case 2 : The compulsive salt eater}

This was a 10-year old girl who had five hospital admissions in four years for investigation of recurrent facial puffiness, abdominal distension, reduced urine output and shortness of breath. She was initially diagnosed as angioneurotic oedema and treated with prednisolone for a short period, without much benefit. She had a habit of taking table salt (sodium chloride) on her palm and licking it. She would defiantly seek out salt, and look for foods and snacks with a high salt content. When a physical reason for her behaviour was not found, she was referred for assessment of her psychological state. This girl was observed to have marked hyperactivity, impulsiveness and attention deficit. She also had frequent anger outbursts and sometimes became physically aggressive. Her early development was normal but she had a moderate degree of reading and spelling disability. On treatment with methylphenidate, the desire for salt completely disappeared. Since then she has had two relapses which resulted from stopping medication. Reintroduction of methylphenidate produced remissions.

\section{Case 3 : The case of alleged sexual abuse}

This 3-year old girl was seen at a general hospital when she developed an offensive and profuse vaginal discharge. Her parents were informed by the hospital staff that they suspect sexual abuse as the cause. The medical specialists made the decision to transfer the child to a tertiary care setting for forensic investigations. For the parents, this was shocking news as they believed the child was well cared for and protected. Subsequent examination revealed a ball of coloured tissue paper inside the child's vagina, which had caused infection and discharge. The parents were referred for psychological help as they were thoroughly shaken by their experience. The mother reported that the child always needed close watching because of her excessive level of activity. She was prone to injury, often had falls and had bruises on her body. A fortnight before, she was admitted to hospital for removal of a bead from inside her nostril. The child was observed to be hyperactive and seemed unable to understand about safety when she scrambled onto heights. No other developmental disorders were identified.

\section{Case 4 : The case of alleged physical abuse}

This 9-year old boy was admitted to hospital with chest pain, body aches, headache and burning sensation on passing urine, but an organic cause was not found. He was also noticed to have multiple scratch marks on face and neck. In addition, there were healed burn marks on the upper abdomen. The possibility of harsh physical punishments was considered and the somatic symptoms were attributed to emotional distress. His social situation too may have contributed towards this assumption by the doctors. His parents separated when he was 5 years old, and he was subsequently looked after by his father and the paternal grandmother. He had lost contact with his mother who had settled down with another man and raised a family. One year ago, he came to live at a Pirivena as his father left to seek employment abroad, and the grandmother could not support him. The child could not give a reasonable explanation about the scratch marks on the neck. However, the healed burn marks on the abdomen according to the child were accidental. He had spilt boiling water on himself by trying to pour it out from a pan. His grandmother confirmed this story. She also added that the child's persistent hyperactivity, uncooperative behaviour and avoidance of schoolwork were the reasons for admission to the Pirivena. 


\section{Discussion}

The presenting complaints of these four children were unusual for ADHD, but compatible with its cardinal features. The repetitive and purposeless sipping of water and licking salt are equivalent to fidgetiness and inability to stay still because of hyperactivity, and difficulty in responding to consequences consequent to impulsiveness. In addition, the low frustration tolerance commonly seen in ADHD may have led to defiance and resistance to intrusion on these behaviours by adults. Hence, it is understandable that remission of all these behaviours occurred with drug treatment for ADHD and relapsed when it was stopped. In the two children who were mistakenly thought to have been abused, impulsiveness and hyperactivity would explain the self-harming behaviours. Mentally retarded children are known to insert or ingest foreign bodies $[5,6]$ but none of the children described could be placed in that diagnosis. Also, in all cases of vaginal discharge in pre-pubertal children, investigating for a foreign body is recommended [7]. No studies on the behavioural or psychiatric aspects in children who insert foreign bodies, compulsively drink water or eat salt were found on literature survey.

\section{Conclusions}

The children discussed here showed potentially lifethreatening behaviours as a result of ADHD. Awareness of such associations is important so that relevant information is obtained and premature and inaccurate medical judgments are avoided.

\section{References}

1. American Psychiatric Association Diagnostic and Statistical Manual of Mental Disorders. 4th ed. Washington DC: American Psychiatric Association, 1994.

2. Hoare P, Beattie T. Children with attention deficit hyperactivity disorder and attendance at hospital. European Journal of Emergency Medicine 2003; 10: 98-100.

3. DiScala C, Lescohier I, Barthel M, Li G. Injuries to children with attention deficit hyperactivity disorder. Pediatrics 1998; 102: 1415-21.

4. Thomas CR, Ayoub M, Rosenberg L, Robert RS, Meyer WJ. Attention deficit hyperactivity disorder \& pediatric burn injury: a preliminary retrospective study. Burns 2004; 30: 221-3.

5. Ramadan N, Pandya NA, Bhaduri B. A Rapunzel with a difference. Archives of Diseases of Childhood 2003; 88: 264

6. Kalan A, Tariq M. Foreign bodies in the nasal cavities: a comprehensive review of the aetiology, diagnostic pointers, and therapeutic measures. Postgraduate Medical Journal 2000; 76: 484-7

7. Smith YR, Berman DR, Quint EH. Premenarchal vaginal discharge: findings of procedures to rule out foreign bodies. Journal of Pediatric and Adolescent Gynecology 2002; 15: 227-30.

\section{Recommendation regarding treatment of participants of ìcontrol groupsî}

Researchers and sponsors have an obligation to provide members of a control group with an established effective treatment whether or not that treatment is and will continue to be unavailable in the country where the research is conducted.

Draft document, US National Bioethics Advisory Committee. 